\title{
Measuring the Complete Transverse Spatial Mode Spectrum of a Wave Field
}

\author{
Gabriel F. Calvo, ${ }^{1}$ Antonio Picón, ${ }^{1}$ and Roberta Zambrini ${ }^{2}$ \\ ${ }^{1}$ Grup de Física Teòrica, Universitat Autònoma de Barcelona, 08193 Bellaterra (Barcelona), Spain and \\ ${ }^{2}$ Institute for Cross-Disciplinary Physics and Complex Systems, \\ IFISC (CSIC-UIB), Palma de Mallorca, 07122, Spain
}

(Dated: December 12, 2007)

\begin{abstract}
We put forward a method that allows the experimental determination of the entire spatial mode spectrum of any arbitrary monochromatic wave field in a plane normal to its propagation direction. For coherent optical fields, our spatial spectrum analyzer can be implemented with a small number of benchmark refractive elements embedded in a single Mach-Zender interferometer. We detail an efficient setup for measuring in the Hermite-Gaussian mode basis. Our scheme should also be feasible in the context of atom optics for analyzing the spatial profiles of macroscopic matter waves.
\end{abstract}

PACS numbers: 42.25.-p, 42.30.-d, 42.15.Eq, 39.20.+q

The temporal [1], vectorial [2] and spatial [3] degrees of freedom of electromagnetic fields with increasing complexity are exploited in applications ranging from communications to medicine. The detailed knowledge of the spatial mode spectrum of these beams, generally emitted by laser devices, is fundamental in optimizing applications as well as in research, both in classical and quantum optics. Many imaging techniques, for instance, are based on the possibility to determine transformations induced by optical elements by knowing the effects on the spatial spectral component of generic signals [4]. Recently, an intense research activity on multimode light has burgeoned in quantum information, communication and imaging [5], with successful demonstrations spanning from nanodisplacement measurements [6], to parallel information [7] and high-dimensional entanglement [8]. The use of transverse multimode beams demands, indeed, the development of techniques allowing to characterize their spatial spectrum and to access the information encoded in different components. Fourier modes are of immediate access through a basic lens set-up [4], while rotating elements allow to determine 'helical' spectra [9]. To the best of our knowledge, however, no direct method is known to measure the Hermite-Gaussian (HG) modes spectrum. Building on the symplectic formalism to describe firstorder optical transformations, we present in this Letter a general strategy to find an arrangement of refractive elements that enables the quantitative measurement of the transverse spatial spectrum of light beams by using a single Mach-Zender interferometer. Due to the generality of our approach, different experimental set-ups can be implemented to determine the full spatial spectrum of multimode transverse beams in different basis, including Laguerre-Gaussian (LG) modes. Here we focus on the spatial spectrum analyzer for HG modes, as these are the most common in laser physics and appear naturally in devices where astigmatism, strain or slight misalignment drive the system toward rectangular symmetry [10]. Furthermore, the presented framework is rather suggestive of analog measurements for matter waves spatial spectra.
Spatial modes are ubiquitous. Sets of modes $u$ satisfying the wave equation $\left(i \partial_{\eta}+\partial_{x}^{2}+\partial_{y}^{2}\right) u=0$, describe broad classes of physical systems. When the evolution variable is $\eta=2 k z$, the sets comprise the optical paraxial modes [10] with wave number $k$ propagating along the $z$ direction. For $\eta=2 m t / \hbar$, the sets model instead the quantum dynamics of free particles of mass $m$ in the $x y$ plane. Since there are two transverse spatial variables, each entire set of mode solutions $u_{m, n}$ is labeled by two integers $m, n$. Relevant examples are the HG and LG mode bases. Any scalar field $\psi$ obeying the above wave equation can thus be decomposed in terms of these modes as $\psi=\sum_{m, n} C_{m, n} u_{m, n}$. A fundamental question then arises: How can one measure the mode spectrum (probabilities) $\mathcal{P}_{m, n}=\left|C_{m, n}\right|^{2}$ of any given scalar field?

To answer the above question we apply a symplectic invariant approach [11-13]. Symplectic methods have been used in theories of elementary particles, condensed matter, accelerator and plasma physics, oceanographic and atmospheric sciences and in optics [14]. Central to our work is the recognition that any linear passive symplectic transformation $S$ acting on the canonical Hermitian operators $\hat{x}, \hat{y}, \hat{p}_{x}$, and $\hat{p}_{y}$ (whose only nonvanishing commutators are $\left.\left[\hat{x}, \hat{p}_{x}\right]=\left[\hat{y}, \hat{p}_{y}\right]=i \succsim\right)$, is associated with a unitary operator $\hat{U}(S)$ generated by the following group

$$
\begin{aligned}
& \hat{\mathcal{N}}=\frac{\hat{x}^{2}+\hat{y}^{2}}{2 w_{0}^{2}}+\frac{\left(\hat{p}_{x}^{2}+\hat{p}_{y}^{2}\right) w_{0}^{2}}{8 \lambda^{2}}, \\
& \hat{\mathcal{L}}_{x}=\frac{\hat{x}^{2}-\hat{y}^{2}}{2 w_{0}^{2}}+\frac{\left(\hat{p}_{x}^{2}-\hat{p}_{y}^{2}\right) w_{0}^{2}}{8 \lambda^{2}}, \\
& \hat{\mathcal{L}}_{y}=\frac{\hat{x} \hat{y}}{w_{0}^{2}}+\frac{\hat{p}_{x} \hat{p}_{y} w_{0}^{2}}{4 \lambda^{2}}, \\
& \hat{\mathcal{L}}_{z}=\frac{\hat{x} \hat{p}_{y}-\hat{y} \hat{p}_{x}}{2 \lambda} .
\end{aligned}
$$

Here, $w_{0}$ is the width of the spatial modes $u_{m, n}$ and $\lambda=1 / k$. The set (1) satisfies the usual $\mathrm{SU}(2)$ algebra $\left[\hat{\mathcal{L}}_{a}, \hat{\mathcal{L}}_{b}\right]=i \varepsilon_{a b c} \hat{\mathcal{L}}_{c}(a, b, c=x, y, z)$, with $\hat{\mathcal{N}}$ being the only commuting generator in the group and $\hat{\mathcal{L}}_{z}$ describing real spatial rotations on the transverse $x y$ plane 
(it is proportional to the orbital angular momentum operator along the wave propagation direction [12]). We can thus represent the most general passive unitary operator $\hat{U}(S)$ associated with $S$ by a single exponential of linear combinations of any of the above generators [13] $\hat{U}(S)=\exp \left[-i\left(\phi_{+} \hat{\mathcal{N}}+\Phi_{-} \cdot \hat{\mathcal{L}}\right)\right]$, with real scalar $\phi_{+}$and vector $\boldsymbol{\Phi}_{-}$parameters. We show below how $\hat{U}(S)$ can be implemented using simple optical elements.

The method proposed here relies on exploiting $\hat{\mathcal{N}}$, together with specific combinations of the generators (1b)(1d), to construct two commuting unitary operators from which the associated symplectic matrices and their experimental implementation can be found relatively easy. To this end, let $|m, n\rangle$ denote the (pure) mode states of order $m+n \geq 0$. We first impose that $|m, n\rangle$ be eigenstates of both $\hat{\mathcal{N}}$ and the operator $\hat{\mathcal{L}}_{\theta, \varphi} \equiv \mathbf{u}_{r} \cdot \hat{\mathcal{L}}$, where $\mathbf{u}_{r}=(\cos \varphi \sin \theta, \sin \varphi \sin \theta, \cos \theta)$ may be conceived as a radially oriented unit vector in the orbital Poincaré sphere $[15,16]$. The eigenstates $|m, n\rangle$ depend on the choice of $\theta$ and $\varphi$ and fulfill $\hat{\mathcal{N}}|m, n\rangle=[(m+n) / 2]|m, n\rangle$ and $\hat{\mathcal{L}}_{\theta, \varphi}|m, n\rangle=[(m-n) / 2]|m, n\rangle$. For instance, the eigenvectors of operators $\hat{\mathcal{L}}_{x}$ and $\hat{\mathcal{L}}_{z}$ are the HG and LG modes, respectively $[12,16]$. Measurements of $|\psi\rangle=$ $\sum_{m, n} C_{m, n}|m, n\rangle$ will involve the action of the unitaries $\hat{U}_{\mathcal{N}}=e^{-i \phi_{+} \hat{\mathcal{N}}}$ and $\hat{U}_{\theta, \varphi}=e^{-i \phi_{-} \hat{\mathcal{L}}_{\theta, \varphi}}$, upon variation of parameters $\phi_{+}$and $\phi_{-}$, which can be externally controlled. Notice that $\hat{U}_{\mathcal{N}}$ is connected with the Gouy phase [17], whereas $\hat{U}_{\theta, \varphi}$ describes $\phi_{-}$-angle rotations about $\mathbf{u}_{r}$, thereby changing the mode superpositions.

In order to extract the complete spectrum $\mathcal{P}_{m, n}$ of a coherent electromagnetic scalar field, an optical scheme is further developed. We remark that for quantum mechanical matter waves (e.g. Bose-Einstein condensates), a conceptually similar approach to analyze their spatial structure should currently be feasible by exploiting atom optics: interferometers [18], beam splitters in atom chips [19], focusing and storage in resonators [20], and conical lenses [21]. We use here a Mach-Zender interferometer with built-in refractive components performing $\hat{U}_{\mathcal{N}}$ and $\hat{U}_{\theta, \varphi}$ : sets of spherical and cylindrical thin lenses. For ease of operation, it is desirable to vary $\phi_{ \pm}$in a way that minimizes the displacements of the optical elements. We explicitly show below that all the required transformations in the interferometer can be achieved solely by rotation and variation of the focal lengths of the lenses. Translations of the lenses are not necessary, although it may turn out to be more convenient in certain instances to carry finite displacements in some of them. In any case, the arms of the interferometer always remain fixed.

Detection of the light intensity difference $\Delta I \equiv I_{B}-I_{A}$ at the two output ports (A and B) of the interferometer provides the data that enables the reconstruction of $\mathcal{P}_{m, n}$. One has $\Delta I \propto\left\langle\psi\left|\left(\hat{U}_{\mathcal{N}}^{\dagger} \hat{U}_{\theta, \varphi}^{\dagger} \hat{U}_{C}+\hat{U}_{C}^{\dagger} \hat{U}_{\mathcal{N}} \hat{U}_{\theta, \varphi}\right)\right| \psi\right\rangle$, where $\hat{U}_{C}$ represents the unitary operator for the compensating lens system in the complementary arm of the interferometer. For clarity, let us assume that $\hat{U}_{C}$ comprises a similar lens set as the one for $\hat{U}_{\mathcal{N}} \hat{U}_{\theta, \varphi}$ (we will show later that this assumption can be removed), with equivalent parameters $\phi_{+}^{\prime}$ and $\phi_{-}^{\prime}$. The mode spectrum and the output intensity difference are directly connected by a double Fourier-like transform

$$
\begin{aligned}
\mathcal{P}_{m, n} & \propto \int_{0}^{4 \pi} \int_{0}^{4 \pi} d \phi_{+} d \phi_{-} \Delta I\left(\phi_{+}-\phi_{+}^{\prime}, \phi_{-}-\phi_{-}^{\prime}\right) \\
& \times e^{i(m-n)\left(\phi_{+}-\phi_{+}^{\prime}\right) / 2} e^{i(m+n)\left(\phi_{-}-\phi_{-}^{\prime}\right) / 2}
\end{aligned}
$$

where the proportionality constant equals $\left(16 \pi^{2}\right)^{-1}$ if $m=n=0$, and $\left(8 \pi^{2}\right)^{-1}$ otherwise. Note that $\Delta I$ possess the symmetry properties $\Delta I\left(\phi_{+} \pm 2 \pi, \phi_{-} \pm 2 \pi\right)=$ $\Delta I\left(\phi_{+} \pm 2 \pi, \phi_{-} \mp 2 \pi\right)=\Delta I\left(\phi_{+}, \phi_{-}\right)$. Our proposed scheme nontrivially generalizes that of Ref. [9], aimed to reveal the quasi-intrinsic nature of the orbital angular momentum degree of freedom for scalar waves. There, by means of the measurement $\hat{U}=e^{-i \phi \hat{\mathcal{L}}_{z}}$, implemented with Dove prisms, the azimuthal index spectrum of spiral harmonic modes could readily be accessed. In our case, we explore the entire transverse mode space labeled by the indices $m$ and $n$. Hence, the combined effect of the two nonequivalent measurements $\hat{U}_{\mathcal{N}}$ and $\hat{U}_{\theta, \varphi}$ is crucial, and would allow us to measure, for instance, either the full spectrum of HG or LG modes.

To show an explicit application of the above approach, we proceed with the characterization and design of an optical system that enables the reconstruction of the $\mathrm{HG}$ mode spectrum $\mathcal{P}_{n_{x}, n_{y}}$ of a light beam $\left(n_{x}, n_{y} \geq 0\right)$. In this scenario, one needs to consider the unitary operators $\hat{U}_{\mathcal{N}}$ and $\hat{U}_{\theta=\pi / 2, \phi=0}=e^{-i \phi_{-} \hat{\mathcal{L}}_{x}}$. By resorting to the Stone-von Neumann theorem $[11,12]$, the symplectic matrices $S_{+}$and $S_{-}$associated to these unitaries are

$$
S_{ \pm}=\left(\begin{array}{cccc}
c_{ \pm} & 0 & z_{0} s_{ \pm} & 0 \\
0 & c_{ \pm} & 0 & \pm z_{0} s_{ \pm} \\
-s_{ \pm} / z_{0} & 0 & c_{ \pm} & 0 \\
0 & \mp s_{ \pm} / z_{0} & 0 & c_{ \pm}
\end{array}\right)
$$

where $c_{ \pm}=\cos \left(\phi_{ \pm} / 2\right), s_{ \pm}=\sin \left(\phi_{ \pm} / 2\right)$, and $z_{0}=$ $w_{0}^{2} /(2 \lambda)$ is the Rayleigh range. Here, $w_{0}$ provides the characteristic width of the HG modes in which the input beam is to be decomposed. From matrices $S_{ \pm}$it is then possible to obtain the corresponding integral transforms that govern the propagation (along the $z$-direction) of any input paraxial wave $\psi(x, y)$ traversing each system. These transforms follow from the general Collins integral representation [11] and lead, in our case, to the kernels

$$
\begin{aligned}
K_{ \pm}\left(x, y ; x^{\prime}, y^{\prime}\right) & =\frac{1}{\pi i\left|s_{ \pm}\right| w_{0}^{2}} \exp \left[\frac{-2 i\left(x x^{\prime} \pm y y^{\prime}\right)}{w_{0}^{2} s_{ \pm}}\right] \\
& \times \exp \left[\frac{i\left(x^{2} \pm y^{2}+x^{\prime 2} \pm y^{\prime 2}\right) c_{ \pm}}{w_{0}^{2} s_{ \pm}}\right] .
\end{aligned}
$$

The output wave functions after $S_{ \pm}$thus result from $\psi_{ \pm}(x, y)=\int d x^{\prime} d y^{\prime} K_{ \pm}\left(x, y ; x^{\prime}, y^{\prime}\right) \psi\left(x^{\prime}, y^{\prime}\right)$. Kernels $(4)$ 


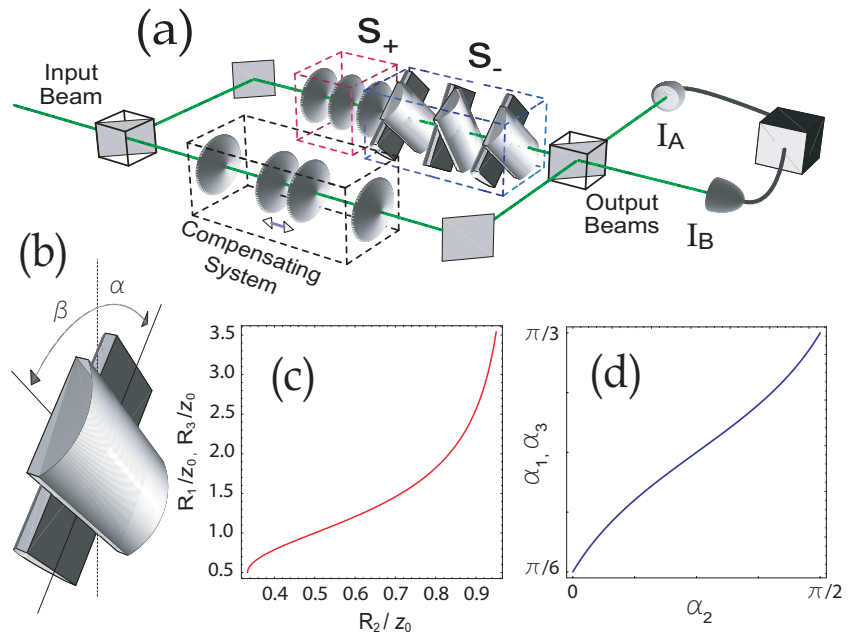

FIG. 1: (Color online) (a) Schematic of the optical implementation to measure the Hermite-Gaussian mode spectrum. Transformations $S_{+}$and $S_{-}$are performed using symmetric sets of three fixed spherical lenses with varying focal lengths and three pairs of rotating cylindrical lenses, respectively. The compensating system involves two settings with four and two spherical lenses, respectively. (b) Detail of one of the assembled pairs of cylindrical lenses required for $S_{-}$. (c) and (d) Operation curves for $S_{+}$and $S_{-}$, respectively (see text).

exhibit the structure of those for the fractional Fourier transform [22]. The singular cases, arising when $s_{ \pm}=0$, can easily be handled too. Integral transforms associated to the unitary operator generated by $\hat{\mathcal{L}}_{y}$ (rather than $\hat{\mathcal{L}}_{x}$ ) have been formulated and lead to the, so-called, gyrator transform [23]. They allow for the full meridian-rotation on the orbital Poincaré sphere and, in particular, for the reciprocal conversion between $\mathrm{HG}$ and LG modes.

The two proposed optical systems for $S_{ \pm}$are symmetric and illustrated in Fig. 1(a). For $S_{+}$, three fixed spherical lenses with varying radii of curvature $R_{j}$ (linked to the focal lengths $f_{j}$ by $R_{j}=\left(\tilde{n}_{j}-1\right) f_{j}, \tilde{n}_{j}$ being the refractive indexes), and placed at equal distances $z_{0}$, are sufficient. They fulfill $R_{1}=R_{3}=z_{0} /\left[1-\cot \left(\phi_{+} / 4\right)\right]$ and $R_{2}=z_{0} /\left(2-s_{+}\right)$. Tunable-focus liquid crystal spherical lenses controlled by externally applied voltages have been demonstrated, displaying a wide range of focal lengths [24]. Figure 1(c) shows the operation curve describing the dependence between $R_{1}, R_{3}$ and $R_{2}$ needed to cover the interval $\pi \leq \phi_{+}<3 \pi$. These values can be attained with the lenses of Ref. [24]. For $S_{-}$, three pairs of cylindrical lenses are required [see Fig. 1(a)]. Each pair of assembled cylindrical lenses is rotated in a scissor fashion with $\alpha=-\beta$ [Fig. 1(b)]. The corresponding angles satisfy $\alpha_{1}=\alpha_{3}=(\pi-\Omega) / 4$ and $\alpha_{2}=\left(3 \pi-\phi_{-}\right) / 4$. The operation curve in Fig. 1(d) represents the variation of the rotation angles in accordance with the constraint imposed by $\cot \left(\phi_{-} / 4\right)=-2 \sin (\Omega / 2)$. This equation is satisfied when $\pi \leq \phi_{-} \leq 3 \pi$ and $-(\pi / 3) \leq \Omega \leq(\pi / 3)$, which lead to $0 \leq \alpha_{2} \leq(\pi / 2)$ and $(\pi / 6) \leq \alpha_{1} \leq(\pi / 3)$.
The radii of curvature of the cylindrical lenses are $R_{1}=$ $R_{2}=R_{5}=R_{6}=z_{0} / 2$ and $R_{3}=R_{4}=z_{0} / 4$. The distance between consecutive pairs is $z_{0} / 2$. With this scheme, the covered values for $\phi_{-} \in[\pi, 3 \pi]$. The fact that both $\phi_{+}$and $\phi_{-}$are restricted to the interval $[\pi, 3 \pi]$, rather than to $[0,4 \pi]$, does not constitute a fundamental limitation. It can be circumvented by employing the compensating system [see Fig. 1(a)] and the symmetry properties of $\Delta I$. If two sequences of measurements are made, each having a different compensating system performing transformations with $\phi_{+}^{\prime}=\phi_{-}^{\prime}=0$ (identity matrix) and $\phi_{+}^{\prime}=0, \phi_{-}^{\prime}=2 \pi$ (minus identity matrix), respectively, then Eq. (2) can be cast $\left(m \rightarrow n_{x}\right.$ and $\left.n \rightarrow n_{y}\right)$ as

$$
\begin{aligned}
\mathcal{P}_{n_{x}, n_{y}} & \propto \int_{\pi}^{3 \pi} \int_{\pi}^{3 \pi} d \phi_{+} d \phi_{-}\left[(-1)^{n_{x}+n_{y}} \Delta I\left(\phi_{+}, \phi_{-}-2 \pi\right)\right. \\
& \left.+\Delta I\left(\phi_{+}, \phi_{-}\right)\right] \cos \left[\frac{\left(n_{x}-n_{y}\right) \phi_{+}+\left(n_{x}+n_{y}\right) \phi_{-}}{2}\right] .
\end{aligned}
$$

The proportionality constant equals $\left(8 \pi^{2}\right)^{-1}$ if $n_{x}=$ $n_{y}=0$, and $\left(4 \pi^{2}\right)^{-1}$ otherwise. The integration intervals in (5) now display the accessible ranges for $\phi_{ \pm}$. The first and second sequences of measurements can be carried out with a compensating system made of four identical and two identical spherical lenses, respectively. By properly choosing their focal lengths, it is not necessary to displace the $S_{ \pm}$systems nor the interferometer arms.

To demonstrate that our data analysis scheme is feasible and does not require a large number of measurements for each $\phi_{ \pm}$, we have numerically simulated the transformation and processing of several input coherent waves. Figure 2 depicts the light profiles entering into the interferometer: an strongly astigmatic Gaussian beam [Fig. 2(a)], an hexapole necklace beam [Fig. 2(d)] and an anisotropic multiring beam [Fig. 2(g)]. Their exact HG mode weight distributions are displayed in the central column of histograms [Fig. 2(b), (e), (h)]. The right column in Fig. 2 represents the weights $\mathcal{P}_{n_{x}, n_{y}}$ from Eq. (5) retrieved after the traversing of the input beams through the system. To simulate the evolution of the various beams, we have used the transformation kernels (4) to calculate the $\Delta I$ in Eq. (5) for 10 different values (in equal increments) per each $\phi_{ \pm} \in[\pi, 3 \pi]$. The reconstructed weights (right column) agree well with the exact weights (central column). In analogy with the WhittakerShannon sampling theorem in Fourier analysis [4], input beams that are mode-band-limited can be exactly reconstructed via our scheme [compare Figs. 2(e) and 2(f)]. That is, if the sampling increments for $\phi_{ \pm}$are smaller than the inverse of the highest contributing mode numbers, reconstruction will be exact. Misalignment of the optical elements is expected to be the main source of errors. The effect on the weights $\mathcal{P}_{n_{x}, n_{y}}$, due to lens displacements (tolerances) $\delta$ with respect to the beam axis, introduces a correction term $\sim\left(\delta / w_{0}\right)^{2}$, which is smaller 

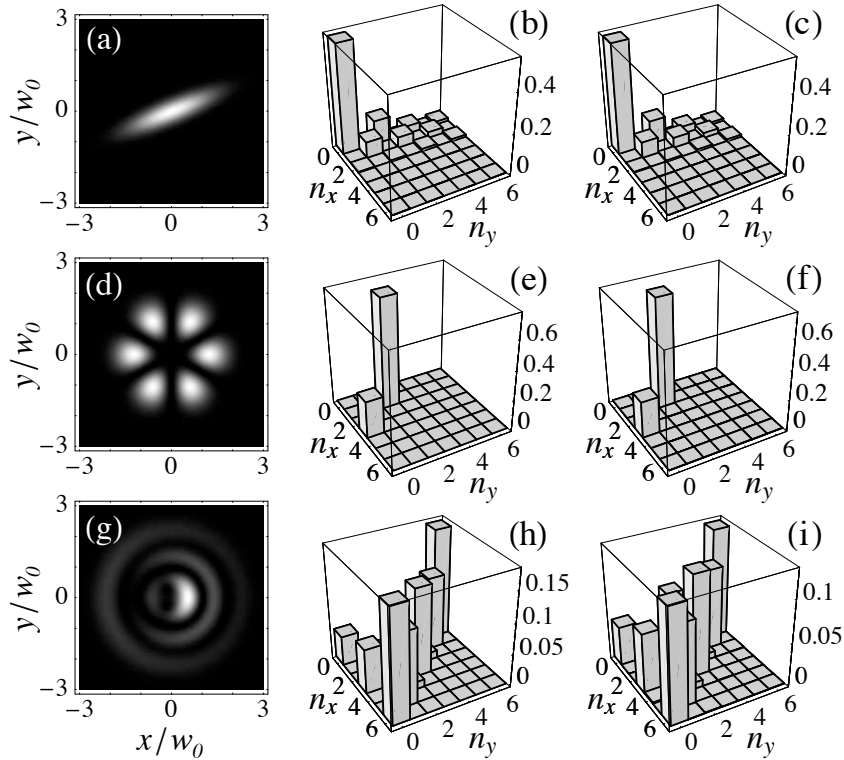

FIG. 2: Mode spectrum analysis. Left column: input beam profiles (a), (d), and (g). Central and right columns: HermiteGaussian mode weight distributions. (b), (e), and (h) exact weights from (a), (d), and (g), respectively. (c), (f), and (i) reconstructed weights $\mathcal{P}_{n_{x}, n_{y}}$ via Eq. (5).

than $1 \%$ for typical values $\delta \lesssim 10 \mu \mathrm{m}$ and $w_{0} \geq 100 \mu \mathrm{m}$.

In conclusion, we have proposed a spatial spectrum analyzer for waves with arbitrary profiles based on a simple set-up of lenses in a Mach-Zender interferometer. The measurement of the modes spectrum of optical beams is fundamental not only to achieve control of laser beam profiles but also to access information encoded in this degree of freedom of the electromagnetic field, in the context of spatial multiplexing. The generality of the presented theoretical analysis encompasses different modes basis and suggests the measurements strategy also for matter waves. The specific set-up for Hermite-Gaussian spectra is presented with details in view of a more immediate experimental realization. Retrieval of the complete spatial mode spectrum of any monochromatic optical field would become an attractive tool for signal analysis and processing when combined with novel holographic recording materials used for modal tailoring and multiplexors. In this respect, amorphous photopolymerizable glasses, exhibiting high refractive index modulation in experimental demonstrations of the optical Pendellösung effect [25], are well suited for this purpose.

We acknowledge financial support from the Spanish Ministry of Science and Technology through Project No. FIS2005-01369, from Govern Balear (PROGECIB-5A), Juan de la Cierva and Ramon y Cajal Grant Programs, and Consolider Ingenio 2010 QIOT CSD2006-00019.
[1] Femtosecond Optical Frequency Comb Technology: Principle, Operation, and Application, J. Ye and S. T. Cundiff Eds. (Springer, 2005).

[2] J. N. Damask, Polarization Optics in Telecommunications (Springer, 2004).

[3] H. H. Barrett and K. J. Myers, Foundations of Image Science (John Wiley \& Sons, 2004).

[4] J. W. Goodman, Introduction to Fourier Optics (Roberts and Company, 2004).

[5] Quantum Imaging, M. I. Kolobov Ed. (Springer, 2007).

[6] N. Treps, N. Grosse, W. P. Bowen, C. Fabre, H.-A. Bachor, and P. K. Lam, Science 301, 940 (2003).

[7] M. Lassen, V. Delaubert, J. Janousek, K. Wagner, H.-A. Bachor, P. K. Lam, N. Treps, P. Buchhave, C. Fabre, and C. C. Harb, Phys. Rev. Lett. 98, 083602 (2007).

[8] A. Mair, A. Vaziri, G. Weihs and A. Zeilinger, Nature 412, 313 (2001); N.K. Langford, R. B. Dalton, M. D. Harvey, J. L. O'Brien, G. J. Pryde, A. Gilchrist, S. D. Bartlett, and A. G. White, Phys. Rev. Lett. 93, 053601 (2004).

[9] R. Zambrini and S. M. Barnett, Phys. Rev. Lett. 96, 113901 (2006).

[10] A. E. Siegman, Lasers (University Science books, 1986).

[11] R. Simon and K. B. Wolf, J. Opt. Soc. Am. A 17, 342 (2000).

[12] G. F. Calvo, A. Picón, and E. Bagan, Phys. Rev. A 73, 013805 (2006).

[13] G. F. Calvo and A. Picón, Phys. Rev. A (to be published); see also quant-ph/07121197.

[14] V. Guillemin and S. Sternberg, Symplectic Techniques in Physics (Cambridge University Press, 1990).

[15] M. J. Padgett and J. Courtial, Opt. Lett. 24, 430 (1999).

[16] G. F. Calvo, Opt. Lett. 30, 1207 (2005).

[17] A. B. Ruffin, J. V. Rudd, J.F. Whitaker, S. Feng, and H. G. Winful, Phys. Rev. Lett. 83, 3410 (1999); J.H. Chow, G. de Vine, M. B. Gray, and D. E. McClelland, Opt. Lett. 29, 2339 (2004).

[18] Atom Interferometry, P. R. Berman Ed. (Academic Press, 1997).

[19] D. Cassettari, B. Hessmo, R. Folman, T. Maier, and J. Schmiedmayer, Phys. Rev. Lett. 85, 5483 (2000).

[20] I. Bloch, M. Köhl, M. Greiner, T. W. Hänsch, and T. Esslinger, Phys. Rev. Lett. 87, 030401 (2001).

[21] S. R. Muniz, S. D. Jenkins, T. A. B. Kennedy, D. S. Naik, and C. Raman, Opt. Express 14, 8947 (2006).

[22] H. M. Ozaktas, Z.Z. Zalevsky, and M. A. Kutay, The Fractional Fourier Transform: with Applications in Optics and Signal Processing (John Wiley \& Sons, 2001).

[23] J. A. Rodrigo, T. Alieva and M. L. Calvo, J. Opt. Soc. Am. A 23, 2494 (2006); J. A. Rodrigo, T. Alieva and M. L. Calvo, Opt. Express 15, 2190 (2007).

[24] H. Ren and S.T. Wu, Opt. Express 14, 11292 (2006); H. Ren, D. W. Fox, B. Wu, and S.T. Wu, Opt. Express 15, 11328 (2007).

[25] M. L. Calvo, P. Cheben, O. Martínez-Matos, F. del Monte, and J. A. Rodrigo, Phys. Rev. Lett. 97, 084801 (2006). 